\title{
From Cells as Computation to Cells as Apps
}

\author{
Andrea Bracciali ${ }^{1}$, Enrico Cataldo ${ }^{2}$, Luisa Damiano ${ }^{3}$, Claudio Felicioli ${ }^{4}$, \\ Roberto Marangoni ${ }^{2}$, and Pasquale Stano $^{5}$ \\ 1 Stirling University, UK abb@cs.stir.ac.uk, \\ 2 University of Pisa, Italy \{enrico.cataldo, roberto.marangoni\}@unipi.it \\ 3 University of Messina, Italy luisa.damiano@unime.it \\ 4 Fabnami, Switzerland pangon@gmail.com \\ 5 Roma Tre University, Rome, Italy pasquale.stano@uniroma3.it
}

\begin{abstract}
We reflect on the computational aspects that are embedded in life at the molecular and cellular level, where life machinery can be understood as a massively distributed system whose macroscopic behaviour is an emerging property of the interaction of its components. Such a relatively new perspective, clearly pursued by systems biology, is contributing to the view that biology is, in several respects, a quantitative science. The recent developments in biotechnology and synthetic biology, noticeably, are pushing the computational interpretation of biology even further, envisaging the possibility of a programmable biology. Several in-silico, in-vitro and in-vivo results make such a possibility a very concrete one. The long-term implications of such an "extended" idea of programmable living hardware, as well as the applications that we intend to develop on those "computers", pose fundamental questions.
\end{abstract}

Keywords: Synthetic biology, Programmable biology, Living hardware

\section{Introduction}

Biology is being understood also as a quantitative and computational science. Distributed information processing and the quantitative dynamics of massively distributed molecular systems are key when investigating life systems. Current breakthroughs in synthetic biology bring into play the possibility of programming such computational biological machineries. In this paper, we reflect on these recent ideas and the perspectives they open. As usually happens in interesting and developing fields, open challenges largely outnumber answers.

The pioneer work of Mendel (1822-1884) established that the phenotype of an organism is determined by quantitative and statistically predictable interactions among "atomic" entities called genes. Genetics and genomics then spawn from this result, which represents a first attempt to describe biological organisms in terms of a network of interactions between elementary entities. Interestingly, this result does not rely on the knowledge of the actual underlying biochemical machinery, but only on the knowledge of information processing at a higher level of abstraction, a well-established approach when studying computation.

The final publication is available at Springer via http://dx.doi.org/10.1007/978-3-319-47286-7_8 
More recently, there has been a transition from a reductionist to a systemic approach. Modern genomics, proteomics, metabolomics and other -omics disciplines, have greatly improved our knowledge about the biochemical and functional network that regulates the cellular life. Many functions are still to be understood, but we have gained strong insights on fundamental components, such as control structures, e.g., power-law in genetic control networks, [6], and important links between network topology and functional aspects, e.g., Flux Balance Analysis studies, [36]. The Human Genome Project [http://www.genome.gov] has contributed to change how biologists view and practice biology. the idea that biology is an informational science with three major types of information emerged: the digital or one-dimensional structure of DNA and genes; the threedimensional structures of proteins, the molecular machines of life; and biological systems as computational systems, which, differently from standard computers, are characterised by producing emergent behaviour. Intuitively speaking, emergent behaviour or properties can only be observed at system level as a result of the interaction, cooperation perhaps, of the system components, and can hardly be understood by observing the components in isolation, nor be deduced by their individual properties. One example is morphogenesis: how can the "program" that allows a beating heart to be formed, be recognised in the single cells that compose it? Emergent behaviour marks a distinguishing feature of life systems versus computer systems, structural decomposition being a desired and sought property of computers and engineered systems.

Quite recent progresses in biotechnology and synthetic biology make such a paradigm move a step forward by bringing into play the programming aspect of computation. The theoretical possibility to control the cellular molecular machinery leads us to imagine programmable devices based on biological cells. This is one of the new frontiers of biology studies, where computer science, engineer, physics and affine disciplines work together to design new organism or protocells able to perform targeted tasks in their biological environment. Beyond the scientific and technical challenges of such an endeavour, current trends also have an impact on philosophical, ethical and social aspects of our society. It is important to remark that the limits and long-term implications of such a scenario are at the moment fully to be understood.

In the following we will discuss and survey some of what we believe to be the most interesting and promising current proposals and possible future scenarios.

\section{Cells as Computation}

Striking results in genomics have largely contributed to the view that cellular biology is a systems science: the complete knowledge of the DNA structure is not enough to describe its metabolic and regulatory functionalities, which depend on the whole cell state and its environment. Such systems are computational: "Many proteins in living cells appear to have as their primary function the transfer and processing of information. ... [they are organised] into biochemical 'circuits' that perform a variety of simple computational tasks, including amplification, 
integration and information storage. The imprint of the environment on the concentration and activity of many thousands of proteins in a living cell ... is like a 'random access memory' containing ever-changing information about the cell's surroundings." [9].

Also, the systems of interest can only be suitably described at a systemic level. It appears therefore quite natural to try to exploit the abstractions used to describe existing computational systems: "To understand biology at the system level, we must examine the structure and dynamics of cellular and organismal function, rather than the characteristics of isolated parts of a cell or organism. ... many breakthroughs in experimental devices, advanced software, and analytical methods are required." [32]. Also, from the Cells as computation paper: "We believe that computer science can provide the much-needed abstraction for biomolecular systems. ... the 'molecule-as-computation' abstraction, in which a system of interacting molecular entities is described and modelled by a system of interacting computational entities. Abstract computer languages ... enable simulation of the behaviour of biomolecular systems ... supporting qualitative and quantitative reasoning on these systems' properties." [52].

A large number of modelling languages and frameworks, often embedding reasoning capability on system behaviour, have been proposed. These include formal languages to describe molecular interaction as communicating computational distributed system and accounting for bio-features such as membranes and compartimentalised systems, e.g. [51,13]; stochastic aspects of molecular interaction, e.g. $[47,16]$; rule-based systems, e.g. [22,11,18]; and Petri-Net based models, a formalism born in Bio-chemistry and widely used in computer science, see the survey [5]. See, e.g., [19] and references therein for further details on such modelling frameworks, whose detailed description is here out of scope. The effort of providing refined modelling and verification frameworks is still ongoing, see. e.g., [46] on including the notion of space for reasoning on non-homogeneous spaces, so overcoming the so-far mainstream assumption of homogeneous reaction environments.

Further research directions have spawned from the approach, examples being the development of DNA-based technology for implementing the computational core of sensing and control processes of synthetic biological components [15], and experiments on building RNA-based logical gates as the basis for the development of "in vivo logic processing" [53].

\section{Towards Programmable Cells}

While the modelling and formalisation of biological systems is still a challenge in mathematics, engineering, and bioinformatics, it is however evident that the development of novel practices in biology laboratories has the potential to support a transition from cell as computation to cell as apps, i.e., from computational to programmable biology. This is the reign of synthetic biology (SB).

SB uses biological parts, devices and systems, e.g., molecules, molecular systems and networks, and (cellular) organisms, for engineering novel synthetic sys- 
tems. Such systems behaves as applications that are running in their execution environment, for instance exploiting the underlying computational capability of molecules. The recent emergence of SB (mainly pioneered by US bioengineers) is based on the availability of powerful bio-analytical technique, especially highthroughput ones, the progress in synthetic capability (synthesis of genes), and the attitude of a young generation of scientists toward the blending and the convergence of biology and engineering.

One of the reasons why SB has become very popular in the last years is that it promises endless possibilities of manipulating organisms for achieving a predefined function [21], although to date only microorganisms have been approached. Current and envisaged applications regard the production of biofuel, chemical intermediates for pharma-chem industry, destroying pollutants, drug development and delivery, diagnosis and therapeutic tasks, e.g. recognise and destroy cancer cells, and so on.

\subsection{Synthetic Biology: Top-down and Bottom-up Approaches}

Despite the term synthetic biology was coined by Leduc in 1912 [34], it is only thanks to recent trends in biological research, combining advancements in genetic manipulation, bioengineering and mathematical modelling, that a new flourishing interest grew around this frontier research field. As a consequence of this recent biotechnological progress, SB is currently seen as one of the most exciting and promising scientific arena, which has attracted scientists with different specialities, bioengineers, and also industrial parties. A possible definition of SB is the following: $S B$ is the design and construction of new biological parts, devices, and systems; and the re-design of existing, natural biological systems for useful purposes (syntheticbiology.org). Useful purposes include (1) the production of any high-value compound from microorganisms (e.g., bio-fuel, drugs, high-value compounds for chemical industry, etc.); (2) the use of microorganisms for special non-medical applications (e.g., bioremediation, fermentation, hybrid living-organic or living-inorganic material, biosensoring); (3) applications in medicine (synthetic cells for attacking cancer cells, or infectious bacteria, virus).

Traditionally, SB operates according to a top-down approach: target functions to be implemented by biological tools (a part, a device, a whole cell) are firstly designed, and then the desired behaviour is obtained by a bioengineering approach, which assembles the tools within a suitable system. This view ultimately relies on the possibility of constructing biological tools just by designing and assembling "standard" biological parts (parts.igem.com) and bio-bricks, just like electronic engineering designs and builds tools starting from components $[37,21]$. Similarly to the electronic chassis, the cell with its core set of geneticmetabolic circuitry typically represents the biological chassis, where parts are implanted and operate according to a pre-defined program, at least in principle.

Although this view suffers of evident limitations (e.g., does not consider explicitly the role of emergent properties in complex systems, and in particular in living systems), it strikingly marks SB. Concepts such as standardisation, optimisation, modularity, orthogonalisation (eliminating between-parts interferences) 
have been introduced and implemented. All this is accompanied by mathematical modelling for the validation and correction of the new genetic-metabolic circuits. Besides being top-down from the viewpoint of design, mainstream SB is also top-down from the operational viewpoint, because it typically starts from living cells, and, via genetic manipulations, or by genome transplantation, transforms them to "synthetic" cells. A remarkable and successful example of such enterprise has been recently reported by Craig Venter's team, which succeeded in transplanting an artificial genome (designed a priori) into a biological receiver cell that obeyed to the new genome commands and proliferated accordingly [24].

Following these recent and high-impact results the scientific community divided among SB-enthusiasts and those that were more cautious with respect to this new technology, which can potentially bring about the construction of dangerous disease-inducing novel organisms.

Clearly, the idea of constructing a synthetic cell that can be programmed to do something useful is very attractive, and it parallels the idea of applications, or apps, running on more traditional computational devices, such as computers and smartphones. Here, the cellular genetic-metabolic network is the chassis for installing a specific program (which is itself a genetic part which is recognised by the cell as a substrate for molecular operations, and at the end obtain a specific behaviour or pattern (e.g., producing a drug, or become fluorescent when a certain substance is present in the environment, etc.).

It must be recalled, however, that despite the straightforward analogies hardware $\leftrightarrow$ cell and software $\leftrightarrow$ genes (or functional components) are strong and widespread in SB, the peculiarity of "living computer" points exactly to the opposite concept of inseparability of biological parts, which evolved together, perform multiple interactions, and loose meaning when transplanted on a too different environment. Moreover, in contrast to electronic circuits, where wires guide electrons, every biological parts potentially interact with all the others in an unpredictable manner, hence undermining the possibility of modular design: adding a functional component to a system may break the component and also system functionality, or, alternatively, the functionality of a component is strongly dependent on their execution environment (a property that is clearly identified as undesirable and dangerous in software engineering). Finally, biological systems are characterized by emergent properties, which as explained above, mark a difference with traditional computers.

In addition to the described top-down perspectives, a new wave in SB embraces research focused on adopting SB practices as a novel approach for generating knowledge, the so-called understanding-by-building. This exciting possibility stems from the fact that SB offers, in principle, the possibility of constructing simplified cells (or even "minimal cells") that might still be functional, but obtained after a strong reduction of biological complexity, so that their behaviour, including emergent patterns, can be ultimately understood thanks to careful experimental observation and mathematical and computational modelling.

Although minimal cells could be obtained, possibly, following a top-down approach, it is the opposite route that is particularly interesting and a true 

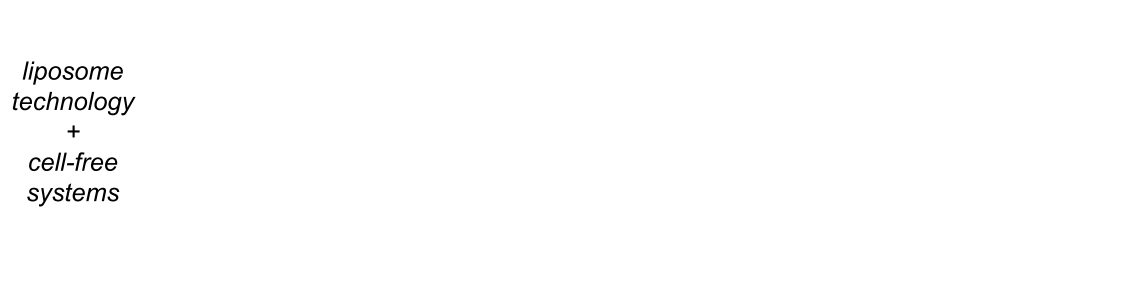

Fig. 1. SSMCs. (a) Biochemical compounds, e.g., DNA, enzymes, ribosomes, tRNAs, small molecules, are encapsulated within liposomes, i.e. hallow spherical microcompartments made by a lipid membrane. (b) "Giant" liposomes: membrane appears red and the vesicle lumen green (confocal laser scanning microscope).

novelty even within SB. This is the laboratory assembly of synthetic, or, more precisely, semi-synthetic [39], minimal cells (SSMCs), generally acknowledged as the bottom-up route. According to the understanding-by-building paradigm, the methodology that is currently under development aims at constructing minimal cells working as physical model to test some specific cellular functions, for example isolating a particular pathway or module from the intricate and indecipherable genetic-metabolic maze which characterises the whole cell.

\subsection{Semi-synthetic Minimal Cells (SSMCs)}

According to the so-called semi-synthetic approach [39] sets of biological macromolecules (DNA, ribosomes, enzymes) are encapsulated inside liposomes, i.e., bubbles made of a lipid membrane, generating SSMCs, which can be designed to produce proteins [11], and then perform biological-like functions (Fig. 1). Thanks to the powerful self-assembly properties of lipids, and the resulting encapsulation of free solutes, cell-like systems emerge spontaneously, with minimal manipulation needs, although study aiming at optimising this process and preparing population of homogeneous SSMCs are ongoing. SSMCs can currently produce simple water-soluble functional proteins and some membrane proteins, which are often required for sensoring purposes, e.g. detecting molecules outside cells and trigger internal responses (see $[54,29,8]$ and references therein).

The design and construction of SSMCs by the modular assembly of genetic, regulatory or metabolic, circuitry in order to specifically exhibit some computation capabilities, presents some potential advantages (1) SSMCs are built from a minimal number of components, facilitating the modeling and the understanding of their dynamic behavior; (2) background and potentially interfering processes (always present in natural cells) can be eliminated in SSMCs; and (3) the synthetic cell behavior is predictable and programmable because the interaction among their components can be designed a priori [3].

Very recent work also shows first steps toward multi-cellular systems. For example, it has been reported that lipid compartments can form clusters following simple adhesive forces $[28,14]$. Protein synthesis is still possible in such assemblies [27], and the idea of prototissue has been put forward, as supported by some pioneer work on membrane-to-membrane connective mojeties $[49,42]$. 
SSMCs originated from studies on the origin of life [45], but actually can now be considered an à la carte design and construction of simplified, not necessarily living, cells for different applications. Autopoiesis, the key concept underlying all minimal cells, is a theory developed by Maturana Varela in the 70s [40,38]. It deals with the basic question "what is life?" and relies on two main hypotheses: (1) The distinctive property of living systems is their autopoiesis, i.e., the capability of producing and maintaining their material identity (themselves) by producing their own components (metabolism); and (2) autopoiesis is a global property: it does not rely on physico-chemical components taken separately, but on the organisation of these components within living systems. In this sense, autopoiesis implies a strong link among synthetic cells and the concept of embodiment, connecting minimal life with minimal cognition, and emergent properties, as further discussed in Section 4.2.

According to the autopoietic theory, the required molecules for constructing a living minimal cell are only those needed for sustaining the self-production of all cellular components, membrane included. Recent genomic analysis of small microorganisms (endosymbiont living inside other cells) shows that about 200 genes suffice, constituting a minimal genome [25]. For non-living systems, less genes are required, depending on the type of functions to be implemented.

Despite these advancements, the ultimate goal of constructing living synthetic cells is still far to be achieved.

Which computational power do SSMCs and other similarly engineered microorganisms have? They share with natural cells the capability of "chemical computing", i.e., processing information by manipulating chemical "signals". Chemical computing comprises molecular recognition, transformation, as well as some control activities, e.g., activation and inhibition of pathways, that largely resound constructs in traditional programming languages. The question about the relationship between biochemical computation and Turing completeness has attracted a lot of interest, e.g., [12], and [59,60] on the Turing universality of DNA computing by reduction to grammars systems and specific classes of cellular automata. Several results prove universality of bio computation, others strive to define suitable theoretical model for what appears to be more suitably described as a reactive and distributed system, than a conventional Turing machine. Parallel cellular automata can be an example of such models. Moreover, probabilistic and stochastic phenomena play an essential part in bio computation, calling for models that account for that. Markov chain based models and the approach developed by Gillespie [26] to describe biochemistry are noticeable examples.

\section{Bio Apps: What Computation?}

The several similarities between Turing computation and Bio computation should not lead to wrong conclusions. Not surprisingly, Apps and BioApps behave differently. We will discuss in the following three fundamental aspects, viz. emergent behaviour, embodiment and the possibility of a Turing test for communicating cells, which can help in such a comparison. 


\subsection{Emergent Behaviour}

Among emergent behaviour, a notably example is given by morphogenesis. In an influential paper [57], Alan Turing suggested that a system of intercellular reaction-diffusion molecules, i.e., of interacting chemical substances diffusing through a tissue, could underlie morphogenesis. Starting from a homogeneous chemical system, some structures can emerge, activated by random inputs, due to the instability of the chemical complex itself. Six emerging spatiotemporal chemical structures could be generated and one of these could underlie differentiation. Turing' s thesis has been recently confirmed on experimental ground by [56], for homogeneous and heterogeneous systems. It is worth mentioning that morphogenesis is at the basis of regenerative medecine: "Regeneration recapitulates in part embryonic development and morphogenesis" from [4]. Morphogenesis is an emergent phenomenon in the sense that it cannot be understood by considering the several part of the system in isolation but only as a result, not even predictable, of the interaction of all the components.

\subsection{Embodiment}

As it has been evidenced before, while the current methodology for synthetic cell construction is based on modern SB, and in particular to the convergence between liposome technology and cell-free systems, the theory of autopoiesis shapes, as a theoretical framework, the long-term goal of constructing a living synthetic unit in the laboratory.

Synthetic cells aim to be, therefore, molecular implementations of autopoietic (self-producing) systems whereby the internal (spatial and dynamic) organization not only accounts for the production of all components, as required from a purely biochemical balance (and maintenance of a topological unit in the space as well), but it is also a pre-requisite for displaying minimal cognitive features.

Autopoietic synthetic units, if and when they will be realised, will perform minimal cognitive dynamics. According to the autopoietic theory, here cognition is viewed as the capacity of an autopoietic system of entering in and maintaining a relationship of structural coupling with its environment, and thus facing and adapting to external changes by performing internal (metabolic) changes without loss of its autopoiesis. In other words, the system will be a cognitive system at a minimal complexity level, and this will be realized fully by the synthetic molecular implementation of the minimal autopoietic organization.

Synthetic cells, realised as minimal autopoietic synthetic units, might be the first fully embodied minimal cognitive systems produced in the laboratory, and for this reason very significant in the field of Embodied Artificial Intelligence (EAI). Synthetic autopoietic units could be able to respond to external input stimuli by sensory-motor coupling, by integrating and compensating the perturbations that can be accommodated in the autopoietic organisation. Interaction with the environment, and with other autopoietic (synthetic or natural biological) units, would might lead to the emergence of new behaviours, among which 
behavioural coupling and communicative interactions. The meaning of the latter would not be defined a priori, but generated from the autopoietic system.

The experimental attempts of constructing autopoietic synthetic units are then relevant for three main reasons: (1) to further understand the autopoietic and cognitive aspects of biological systems and, in particular, biological cells, providing a theoretically grounded and fully tuneable model based on molecular embodiment; (2) to study AI in a more biologically-rooted way at a level of minimal complexity; (3) to move forward more complex systems such as hybrid bio-mechanical robots and, more in general, intelligent systems according to a radical embodiment perspective.

From a more general viewpoint, the development of synthetic cells realised as minimal autopoietic units points at enforcing a paradigm shift from the traditional computationalist cognitive science (cognition as elaboration of exogenous information) to the embodied one (cognition as generation of internal meanings for external events), and therefore understanding first, and developing later, systems capable of adaptive responses to the wide bandwidth of physical communication channel by means of patterns of sensory modalities.

\subsection{A Turing Test for Communicating Cells}

The growing ability of synthetic biologists to realise synthetic cells capable of encode, send, receive and decode chemical signals, translates in both practical and conceptual advancements.

From the practical viewpoint, as remarked in Section 3.2, these advancements might lead to synthetic cells capable of communicating with natural cells and pave the way to future smart drug delivery systems. LeDuc et al. [33] put forward a lucid vision of such an application, introducingthe concept of a "nanofactory" to be introduced in the body to convert pre-existing materials into therapeutic compounds, or transform molecules that a patient is unable to process, due to some medical condition, into other compounds that the body can process.

An interesting and somehow provocative conceptual scenario has been devised, on the other hand, by Cronin, Krasnogor, Davis and collaborators in 2006 [17], arguing that a sort of Turing test, based on minimal communication skills, could help to determine whether a system is alive (at the cellular level), bypassing the question of what life is, as the original Turing test was devised to determine whether a system is intelligent, bypassing the problem of defining what intelligence is. The authors describe how such kind of test could help in the field of artificial cellularity, and for the recognition of life in general. In this re-discovered cellular imitation game the setup is such that a synthetic cell must imitate a natural cell. The imitation becomes perfect when a natural cell as interrogator cannot distinguish a natural from a synthetic cell. Even if not explicitly said, the Turing test for synthetic cells would rely on chemical communication.

It is however evident that the Turing test suffers of some limitations. In particular, it allows only a superficial recognition of intelligence. As it has been often remarked, the artificial/synthetic imitation of, or resemblance with, the 
natural-biological partner could only be superficial and exterior, without implying the same generative mechanism. This caveat is particularly evident in attempts of comparing AIs with human intelligence, because it is conceivable a machine that imitates some aspects of human reasoning without sharing with human brain (or better, with the human body) the same experience in terms of autonomy, embodiment, emergence, sense-making. Does a Turing test based on synthetic cells suffer of the same or similar limitations? Due to the intrinsic embodied nature of autopoietic synthetic cells and of their minimal cognitive features, there is a significant difference with a computer simulating the human intelligence, and this difference is due to the intrinsic embodiment of synthetic cell processes. At this minimal level, will the synthetic/artificial cells really reproduce the cognitive pattern of a natural-biological partner? Will the synthetic vs. natural barrier indeed become ill-defined and possibly disappear?

While it is still not possible to reply to the above questions, research is progressing from the experimental side. After the pioneer work of Davis [23], based on a chemical cell (a chell) sending a signal to the bacterium Vibrio harveyi, and the more recent SB approach by Mansy [35], a research program is currently under development in our laboratory based on acyl-homoserine-lactones, which are simple quorum sensing signal molecules used by bacteria [55].

\section{$5 \quad$ Future Perspectives}

In this section we will illustrate some possible directions for the creation of programable synthetic cells and applications in the field of neurochemical processes underlying memory, given their relevance in the human mental health, from traumatic stress disorder to memory disfunctions.

\subsection{Towards Synthetic Cells 2.0}

Although it is easy to imagine how synthetic cells can be constructed, their actual realization in the laboratory is still difficult. As it has been remarked in Section 3.2, the current artificial cell-like systems are quite limited in terms of functions implemented, and, importantly, they are not autonomous with respect to most of the important features, such as energy generation, self-reproduction, control of internal dynamics, and so on. In other words we are still far from constructing a truly autopoietic cell.

It is nevertheless possible to sketch some possible scenarios for the next generation of synthetic cells, maybe as a roadmap for synthetic cells 2.0, at least to recognize what seem, today, the future directions of this field.

First, the development of synthetic cells which could communicate with biological cells is one of the most intriguing aspects. Synthetic cells that are able to produce, manipulate, and respond to biochemical signals in a controllable and programmable way can be powerful tools for new bio-chemical ITCs [44, 43]. This fascinating path can be approached simply by extending current technology to molecular circuitry specifically designed for chemical signalling. Even 
if not alive, communicating synthetic cells could play a role in conceptual and applicative advancements.

Second, the ultimate goal of semi-synthetic minimal cell research is, of course, the creation of a synthetic cell capable of re-producing itself (i.e., all its parts, membrane included) by means of internal mechanisms and without intervention from outside. Such a cell would be autopoietic, but it is an open question whether it would be alive. In contrast with the early position of autopoiesis proponents, for whom an autopoietic system is alive, it has been recently argued that autopoiesis is a necessary yet not sufficient condition for being alive [7].

Third, another interesting research direction, which is currently pursued (Damiano and Stano, in preparation) is the connection between the SB approach and the field of artificial intelligence (AI). Can SB (i.e. synthetic cells) be useful for the "synthetic exploration" of natural cognition, and in particular minimal cognition? Embodied AI is generally concerned with the design and construction of robots, and especially the cybernetic control of movements, by means of sensori-motor coupling. However, working in the biochemical domain - which is the domanin where life actually originated - offers several advantages for developing embodied AI (a sort of bio-chemical AI). This implies a necessary reduction of complexity in AI goals, but on the other hand molecular embodiment can be the only way to approach the natural performances of biological systems. They indeed function by exploiting macromolecules, their interaction with environment and with other molecules, and their structural capability of modify themselves in response to some stimuli. All this means that starting from minimal synthetic cell it would become possible to construct and study minimal autopoietic cognitive systems so to generate a truly embodied AI.

\subsection{Programming Brain Processes}

The high throughput of data in neurobiology have stimulated the development of theoretical models of neural processes. There has been a lot of progress from the publication of the Hodgkin-Huxley model that elucidated the mechanisms of genesis and propagation of the action potential. Since then, models of interneuronal communication, dendritic spines and synaptic boutons dynamics, neuromodulation, plasticity and development have been proposed [50,30].

Neuronal activity can be measured in the living brain and correlated with ongoing behaviour. To overcome correlation and investigate causal impact of neuronal activity to behaviour, new techniques have been developed, controlling single neurons in a living brain with high spatiotemporal resolution: optogenetics let us control neuronal activity in-vivo by using photons, and optogenetics actuators can be placed in specific cell types gaining high spatiotemporal resolution. To program animal behaviour by manipulating neuronal activity has been proved possible but improvements in models and techniques are needed [10].

Synaptic plasticity is understood to be one of the modality underlying learning and memory processes, whose mechanisms, at molecular and cellular level, have been at least in part elucidated during the last decades, as reviewed in 
[31]. Several insights have been obtained on the spatial and temporal distribution of memory, on how many tipes of memory exist and on the molecular and structural mechanisms of some forms of memory. Memories can be classified as explicit memory, also called declarative memory, regarding facts and events and implicit memory, also called nondeclarative memory, regarding perception and motor skills. Another feature of memory is its duration. One can distinguish short-term, intermediate-term and long-term memory.

Biological memory storage is the result of the interaction of several processes. Interestingly, long-term memory can be divided into four stages: learning, consolidation, storage and retrieval [58]. During the retrieval, update and integration of a given memory with other memories, it is possible to obtain destabilization and restabilization of that memory. Depending on the temporal stage of a given consolidated memory, the retrieval can yield a destabilization of the memory traces and a possible change of them $[2,1,41]$. A complex molecular machinery is involved in such memory processes [48]. Neurochemical computation regards not only neuromodulation, homeostasis, development and cellular housekeeping but also pattern detection and memory. It s believed that chemical computation is hundred times the electrical one [6].

Informally speaking, such processes could be seen as a molecular foundation of psychology, which manipulates and heals disturbing memories through recalling them [20]. Can such molecular machine be programmed? Which are the implications?

\section{Concluding Remarks}

We have considered analogies between in-silico and in-vivo computation, according to a consolidated research approach which informs the field of system biology. Furthermore, we have pushed further such an analogy, discussing the possibility of programming computational life systems, which is the goal of the currently strongly developing field of synthetic biology. We have presented current results and open problems of specific approaches in synthetic biology, mainly in the area of semi-synthetic minimal cells. Finally, we have considered both some of the differences between in-silico and in-vivo computation, and pointed out a few possible future developments, such as possible interactions of synthetic and natural cells, and the possibility of programming cells that play a role in brain processes.

This is an overview of a currently happening, multidisciplinary revolution which will transform both computational and life sciences, with impacts on several aspects of our daily life, such as novel therapies, the production of bio-energy and food, to cite but a few. This paper aim to contribute to the diffusion of the scientific knowledge that will be needed to inform related choices on philosophical, ethical and social aspects of such an endeavour.

Acknowledgements. PS and LD thank Pier Luigi Luisi for insightful discussions on Maturana-Varela autopoiesis, cognition, minimal life, and embodiment. 


\section{References}

1. Abel, T., Lattal, K.M.: Molecular mechanisms of memory acquisition, consolidation and retrieval. Curr. Opin. Neurobiol. 11(2), 180-187 (Apr 2001)

2. Alberini, C.M.: Mechanisms of memory stabilization: are consolidation and reconsolidation similar or distinct processes? Trends Neurosci. 28(1), 51-56 (Jan 2005)

3. Altamura, E., Stano, P., Walde, P., Mavelli, F.: Giant vesicles as micro-sized enzymatic reactors: perspectives and recent experimental advancements. International Journal of Unconventional Computing 11, 5-21 (2015)

4. Atala, A., Lanza, R., Thomson, J., Nerem, R. (eds.): Foundation of Regenerative Medicine. Academic Press (2010)

5. Baldan, P., Cocco, N., Marin, A., Simeoni, M.: Petri nets for modelling metabolic pathways: a survey. Natural Computing 9(4), 955-989 (2010)

6. Bhalla, U.S.: Molecular computation in neurons: a modeling perspective. Curr. Opin. Neurobiol. 25, 31-37 (Apr 2014)

7. Bitbol, M., Luisi, P.L.: Autopoiesis with or without cognition: defining life at its edge. J R Soc Interface 1(1), 99-107 (Nov 2004)

8. Blain, J.C., Szostak, J.W.: Progress Toward Synthetic Cells. Annual Review of Biochemistry 83(1), 615-640 (2014)

9. Bray, D.: Protein molecules as computational elements in living cells. Nature 376, 307-312 (1995)

10. Brette, R., Destexhe, A. (eds.): Handbook of Neural Activity Measurement. Cambridge University Press (2012)

11. Calzone, L., Fages, F., Soliman, S.: BIOCHAM: an environment for modeling biological systems and formalizing experimental knowledge. Bioinformatics 14(22), 1805-1807 (2006)

12. Cardelli, L., Zavattaro, G.: Turing universality of the biochemical ground form. mathematical structures in computer. Science. 20, 45-73 (2010)

13. Cardelli, L.: Brane calculi-interaction of bological membranes. In: Danos, V., Schachter, V. (eds.) Proceedings of Computational Methods in Systems Biology. pp. 257-280. No. 3082 in LNCS, Springer, Paris (2004)

14. Carrara, P., Stano, P., Luisi, P.L.: Giant vesicles "colonies": a model for primitive cell communities. Chembiochem 13(10), 1497-1502 (Jul 2012)

15. Chen, Y., Dalchau, N., Srinivas, N., Phillips, A., Cardelli, L., Soloveichik, D., Seelig, G.: Programmable chemical controllers made from dna. Nature Nanotechnology 8, 755-762 (2005)

16. Ciocchetta, F., Hillston, J.: Bio-PEPA: a Framework for the Modelling and Analysis of Biochemical Networks. Theoretical Computer Science 33-34(410), 3065-3084 (2004)

17. Cronin, L., Krasnogor, N., Davis, B.G., Alexander, C., Robertson, N., Steinke, J.H.G., Schroeder, S.L.M., Khlobystov, A.N., Cooper, G., Gardner, P.M., Siepmann, P., Whitaker, B.J., Marsh, D.: The imitation game-a computational chemical approach to recognizing life. Nat. Biotechnol. 24(10), 1203-1206 (Oct 2006)

18. Danos, V., Feret, J., Fontana, W., Harmer, R., Krivine, J.: Rule-based modelling of cellular signalling. In: Procs. CONCUR. LNCS, vol. 4703, pp. 17-41. Springer (2007)

19. Degano, P., Bracciali, A.: Process calculi, systems biology and artificial chemistry. In: Rozenberg, G., Bck, T., Kok, J. (eds.) Handbook of Natural Computing. Springer (2012) 
20. Dudai, Y.: Consolidation: fragility on the road to the engram. Neuron $17(3), 367-$ 370 (1996)

21. Endy, D.: Foundations for engineering biology. Nature 438(7067), 449-453 (2005)

22. Fontana, W., Buss, L.W.: The arrival of the fittest: Toward a theory of biological organization. Bull. Math. Biol. 1(56), 1-64 (1994)

23. Gardner, P.M., Winzer, K., Davis, B.G.: Sugar synthesis in a protocellular model leads to a cell signalling response in bacteria. Nat Chem 1(5), 377-383 (Aug 2009)

24. Gibson, D.G., Glass, J.I., Lartigue, C., Noskov, V.N., Chuang, R.Y., Algire, M.A., Benders, G.A., Montague, M.G., Ma, L., Moodie, M.M., Merryman, C., Vashee, S., Krishnakumar, R., Assad-Garcia, N., Andrews-Pfannkoch, C., Denisova, E.A., Young, L., Qi, Z.Q., Segall-Shapiro, T.H., Calvey, C.H., Parmar, P.P., Hutchison, 3rd, C.A., Smith, H.O., Venter, J.C.: Creation of a bacterial cell controlled by a chemically synthesized genome. Science 329(5987), 52-56 (Jul 2010)

25. Gil, R., Silva, F.J., Peretó, J., Moya, A.: Determination of the core of a minimal bacterial gene set. Microbiol. Mol. Biol. Rev. 68(3), 518-537 (2004)

26. Gillespie, D.T.: Exact stochastic simulation of coupled chemical reactions. The Journal of Physical Chemistry 81(25), 2340-2361 (1977)

27. Hadorn, M., Boenzli, E., Sørensen, K.T., De Lucrezia, D., Hanczyc, M.M., Yomo, T.: Defined DNA-mediated assemblies of gene-expressing giant unilamellar vesicles. Langmuir 29(49), 15309-15319 (Dec 2013)

28. Hadorn, M., Eggenberger Hotz, P.: DNA-mediated self-assembly of artificial vesicles. PLoS ONE 5(3), 9886 (2010)

29. Ichihashi, N., Matsuura, T., Kita, H., Sunami, T., Suzuki, H., Yomo, T.: Constructing partial models of cells. Cold Spring Harb Perspect Biol 2(6), 4945 (2010)

30. Jaeger, D. (ed.): Encyclopedia of Computational Neuroscience. Springer (2015)

31. Kandel, E.R., Dudai, Y., Mayford, M.R.: The Molecular and Systems Biology of Memory. Cell 157(1), 163-186 (2014)

32. Kitano, H.: Systems biology: A brief overview. Science 1(295 (5560)), 1662-4 (2002)

33. LeDuc, P.R., Wong, M.S., Ferreira, P.M., Groff, R.E., Haslinger, K., Koonce, M.P., Lee, W.Y., Love, J.C., McCammon, J.A., Monteiro-Riviere, N.A., Rotello, V.M., Rubloff, G.W., Westervelt, R., Yoda, M.: Towards an in vivo biologically inspired nanofactory. Nat Nano 2(1), 3-7 (2007)

34. Leduc, S.: La Biologie Synthétique. Etudes de Biophysique, A. Poinat, Paris, 1st ed. edn. (1912), http://www.peiresc.org/bstitre.htm

35. Lentini, R., Santero, S., Chizzolini, F., Cecchi, D., Fontana, J., Marchioretto, M., Del Bianco, C., Terrell, J.L., Spencer, A.C., Martini, L., Forlin, M., Assfalg, M., Dalla Serra, M., Bentley, W.E., Mansy, S.S.: Integrating artificial with natural cells to translate chemical messages that direct E. coli behaviour. Nat Comm 5 (2014)

36. Long, C.P., Antoniewicz, M.R.: Metabolic flux analysis of escherichia coli knockouts: lessons from the keio collection and future outlook. Current opinion in biotechnology 28, 127-133 (2014)

37. de Lorenzo, V., Danchin, A.: Synthetic biology: discovering new worlds and new words. EMBO Rep. 9(9), 822-827 (2008)

38. Luisi, P.L.: Autopoiesis: a review and a reappraisal. Naturwissenschaften 90(2), 49-59 (2003)

39. Luisi, P.L., Ferri, F., Stano, P.: Approaches to semi-synthetic minimal cells: a review. Naturwissenschaften 93(1), 1-13 (2006)

40. Maturana, H.R., Varela, F.J.: Autopoiesis and Cognition: The Realization of the Living. D. Reidel Publishing Company, 1st edn. (1980)

41. McKenzie, S., Eichenbaum, H.: Consolidation and reconsolidation: two lives of memories? Neuron 71(2), 224-233 (Jul 2011) 
42. Moritani, Y., Nomura, S.M., Morita, I., Akiyoshi, K.: Direct integration of cellfree-synthesized connexin-43 into liposomes and hemichannel formation. FEBS J. 277(16), 3343-3352 (Aug 2010)

43. Nakano, T., Eckford, A.W., Haraguchi, T.: Molecular Communications. Cambridge University Press, Cambridge UK (2013)

44. Nakano, T., Moore, M., Enomoto, A., Suda, T.: Molecular Communication Technology as a Biological ICT. In: Sawai, H. (ed.) Biological Functions for Information and Communication Technologies, pp. 49-86. No. 320 in Studies in Computational Intelligence, Springer Berlin Heidelberg (Jan 2011)

45. Oberholzer, T., Wick, R., Luisi, P.L., Biebricher, C.K.: Enzymatic RNA replication in self-reproducing vesicles: an approach to a minimal cell. Biochem. Biophys. Res. Commun. 207(1), 250-257 (Feb 1995)

46. Parvu, O., Gilbert, D.R., Heiner, M., Liu, F., Saunders, N., Shaw, S.: Spatialtemporal modelling and analysis of bacterial colonies with phase variable genes. ACM Trans. Model. Comput. Simul. 25(2), 13 (2015)

47. Priami, C., Regev, A., Shapiro, E., Silvermann, W.: Application of a stochastic name-passing calculus to representation and simulation of molecular processes. Theoretical Computer Science 1(325), 141-167 (2004)

48. Ramakrishnan, N., Bhalla, U.S.: Memory switches in chemical reaction space. PLoS Comput. Biol. 4(7), e1000122 (2008)

49. Ramundo-Orlando, A., Serafino, A., Villalobo, A.: Gap junction channels reconstituted in two closely apposed lipid bilayers. Arch. Biochem. Biophys. 436(1), 128-135 (Apr 2005), wOS:000227685000015

50. Reeke, G.N., Poznanski, R.R., Lindsay, K.A., Rosenberg, J.R., Sporn, O. (eds.): Modeling in the Neuroscience - from Biological Systems to Neuromimetic Robotics. CRC Press (2005)

51. Regev, A., Panina, E., Silverman, W., Cardelli, L., Shapiro, E.: BioAmbients: An abstraction for biological compartements. Theoretical Computer Science 1(325), 141-167 (2004)

52. Regev, A., Shapiro, E.: Cellular abstractions: Cells as computation. Nature 419, 343 (2002)

53. Seelig, G., Soloveichik, D., Zhang, D., Winfree, E.: Enzyme-free nucleic acid logic circuits. Science 314, 1584-1588 (2006)

54. Stano, P., Carrara, P., Kuruma, Y., Souza, T.P., Luisi, P.L.: Compartmentalized reactions as a case of soft-matter biotechnology: synthesis of proteins and nucleic acids inside lipid vesicles. J. Mater. Chem. 21(47), 18887-18902 (2011)

55. Stano, P., Rampioni, G., Carrara, P., Damiano, L., Leoni, L., Luisi, P.L.: Semisynthetic minimal cells as a tool for biochemical ICT. BioSys. 109(1), 24-34 (2012)

56. Tompkinsa, N., Lia, N., Girabawea, C., Heymanna, M., Ermentroutc, G.B., Epsteind, I.R., Fradena, S.: Testing turing's theory of morphogenesis in chemical cells. PNAS 111(12), 4397-4402 (2014)

57. Turing, A.M.: The chemical basis of morphogenesis. Philosophical Transactions of the Royal Society of London. Series B, Biological Sciences 237(641), 37-72 (1952)

58. Wang, H., Hu, Y., Tsien, J.Z.: Molecular and systems mechanisms of memory consolidation and storage. Prog. Neurobiol. 79(3), 123-135 (Jun 2006)

59. Winfree, E.: Algorithmic self-assembly of DNA. Ph.D. thesis, California Institute of Technology (1998)

60. Winfree, E., Yang, X., Seeman, N.C.: Universal computation via self-assembly of dna: Some theory and experiments. DNA Based Computers 44, 191-213 (1999) 